\title{
New Boundary Constraints for Elliptic Systems Used in Grid Generation Problems
}

\author{
Upender K. Kaul \\ Computational Sciences Division \\ NASA Ames Research Center \\ Moffett Field, CA 94035
}

\begin{abstract}
This paper discusses new boundary constraints for elliptic partial differential equations as used in grid generation problems in generalized curvilinear coordinate systems. These constraints, based on the principle of local conservation of thermal energy in the vicinity of the boundaries, are derived using the Green's Theorem. They uniquely determine the so called decay parameters in the source terms of these elliptic systems. These constraints ${ }^{1}$ are designed for boundary clustered grids where large gradients in physical quantities need to be resolved adequately. It is observed that the present formulation also works satisfactorily for mild clustering. Therefore, a closure for the decay parameter specification for elliptic grid generation problems has been provided resulting in a fully automated elliptic grid generation technique. Thus, there is no need for a parametric study of these decay parameters since the new constraints fix them uniquely. It is also shown that for Neumann type boundary conditions, these boundary constraints uniquely determine the solution to the internal elliptic problem thus eliminating the non-uniqueness of the solution of an internal Neumann boundary value grid generation problem.
\end{abstract}

\section{Keywords:}

New boundary constraints, Automated elliptic grid generation; Decay parameters, Neumann boundary conditions, Conservation of thermal energy.

\section{Introduction}

A large amount of effort has been devoted to developing, enhancing and using the grid generation capability ${ }^{1-7}$ through the solution of elliptic partial differential equations (pdes). As shown in the present study, elliptic pdes used in grid generation problems reduce to limiting forms near boundaries that are similar to the equations used in nuclear physics, diffusion-reaction problems, vortex problems, electric space charge problems, steady state heat transfer (conduction and convection) through long thin fins, etc. In the grid generation problems, these pdes contain appropriate inhomogeneous terms that control the distribution of grid points especially near the boundaries. In the literature, the elliptic pdes used for grid generation are erroneously referred to as Poisson equations which contain source terms that are functions of only the independent variables, whereas, in the pdes for grid generation, these inhomogeneous terms also contain terms proportional to the dependent variables. Actually, in grid generation problems, close to a curvilinear boundary, the governing

${ }^{1}$ Invention under review for NASA Patent 
equations reduce to the long thin fin heat transfer equations with a finite heat transfer coefficient in the transverse direction (normal to the plane of paper) and a large heat transfer coefficient in the lateral direction.

Most of the elliptic grid generation studies referred to above have been centered on developing body conforming grids around bodies for external fluid flow simulations. The grids thus generated are smooth with at least first two derivatives continuous, appropriately stretched or clustered normal to any given coordinate direction and orthogonal over most of the grid domain. The inhomogeneous terms afford a grid control to satisfy clustering and orthogonality around specific surfaces (in three dimensions) and lines (in two dimensions).

In external flows, these inhomogeneous terms, i.e., the source terms and the dependent variable proportional terms, are designed to vanish away from the body so the problem reduces to solving a Laplacian away from the body.

In the present study, the inhomogeneous terms used are appropriate for an interior grid generation problem where all the boundaries enveloping the grid will affect the solution through these terms. These terms are designed by interpreting their meaning physically through the principle of local conservation of thermal energy close to the grid boundaries.

Two geometrical configurations, an annulus and a gear tooth, are considered to test the new boundary constraints derived in this study. The gear tooth geometry treated here corresponds to that of a planar cross-section of a spiral-bevel pinion gear tooth typical of the OH-58 helicopter transmission pinion. This study is driven by the need to generate time-series vibration signatures from the OH-58 helicopter transmission by finite difference simulation of the appropriate structural dynamic equations. The choice of elliptic pdes for grid generation is entailed by the need to generate time series data as accurately as possible (see relative comparison with other representative grid generation methods in Ref. 7).

\section{Governing Equations} The two-dimensional governing equations for an elliptic grid generation problem in an appropriately
defined planar domain are

$$
\begin{aligned}
\xi_{x x}+\xi_{y y} & =P(\xi, \eta) \\
\eta_{x x}+\eta_{y y} & =Q(\xi, \eta)
\end{aligned}
$$

where $\xi$ and $\eta$ are the generalized curvilinear coordinates, $\mathrm{x}$ and $\mathrm{y}$ are the Cartesian coordinates, and the $P(\xi, \eta)$ and $Q(\xi, \eta)$ are the inhomogeneous terms.

The form of the inhomogeneous terms, $P$ and $Q$, is, e.g., exponential ${ }^{2}$ and is given by

$$
\begin{gathered}
P(\xi, \eta)=-a_{i}(\eta) \operatorname{sgn}\left(\xi-\xi_{i}\right) \exp \left(-b_{i}\left|\xi-\xi_{i}\right|\right) \\
Q(\xi, \eta)=-c_{i}(\xi) \operatorname{sgn}\left(\eta-\eta_{i}\right) \exp \left(-d_{i}\left|\eta-\eta_{i}\right|\right)
\end{gathered}
$$

where $i$ refers to the grid boundary in question.

For the sake of argument, without loss of generality, if we take the case where $\xi>\xi_{i}$ and $\eta>\eta_{i}$ , then we have the inhomogeneous terms as

$$
\begin{aligned}
& P(\xi, \eta)=-a_{i}(\eta) \exp \left(-b_{i}\left(\xi-\xi_{i}\right)\right) \\
& Q(\xi, \eta)=-c_{i}(\xi) \exp \left(-d_{i}\left(\eta-\eta_{i}\right)\right)
\end{aligned}
$$

At the boundaries, where $\xi=\xi_{i}$ and $\eta=\eta_{i}$, Equations (1c) and (1d) respectively become

$$
P\left(\xi_{i}, \eta\right)=-a_{i}(\eta)
$$

and

$$
Q\left(\xi, \eta_{i}\right)=-c_{i}(\xi)
$$

When $b_{i}\left|\xi-\xi_{i}\right|$ or $d_{i}\left|\eta-\eta_{i}\right|$ is small, the inhomogeneous terms take the form given by

$$
P(\xi, \eta)=-a_{i}(\eta)\left(1-b_{i}\left(\xi-\xi_{i}\right)\right)
$$


and

$$
Q(\xi, \eta)=-c_{i}(\xi)\left(1-d_{i}\left(\eta-\eta_{i}\right)\right)
$$

Therefore, the governing equation for, e.g., $\xi$, in the vicinity of the boundary $\xi_{i}$, becomes

$$
\xi_{x x}+\xi_{y y}=-a_{i}(\eta)\left(1-b_{i}\left(\xi-\xi_{i}\right)\right)
$$

or

$$
\xi_{x x}+\xi_{y y}-a_{i}(\eta) b_{i} \xi=-a_{i}(\eta)-a_{i}(\eta) b_{i} \xi_{i}
$$

If the term, $a_{i} b_{i} \xi$, were absent, the resulting equation would turn out to be a Poisson equation. The equation given above arises, e.g., in the steady state heat conduction problems in long thin fins, where $\xi$ is the temperature and where the heat transfer coefficient in the transverse thin direction is moderate but is large in the lateral direction, and there is a balance amongst the heat conducted through the fin, heat carried away from or to it through convection in proportion to this moderate heat transfer coefficient and the heat sources/sinks distributed over the domain. Consider the case when $\xi>\xi_{i}$, then, defining a new variable

$$
\theta=\xi-\xi_{i}
$$

Equation(2) becomes

$$
\theta_{x x}+\theta_{y y}-a_{i} b_{i} \theta=-a_{i}
$$

The term, $-a_{i}$, can be interpreted as a heat source term.

Equation(3) tells us that when $\xi>\xi_{i}$, there is a balance between the heat convected from a control volume in the interior to the boundary $\xi_{i}$, heat conducted out of this control volume and the heat lost from the control volume due to the heat sink, $a_{i}(\eta)$.

Similarly, if we consider the case when $\xi_{i}>\xi$, then Equation (2) becomes

$$
\theta_{x x}+\theta_{y y}-a_{i}(\eta) b_{i} \theta=a_{i}(\eta)
$$

where $\theta=\xi_{i}-\xi$

The term, $a_{i}$, can be interpreted as a heat sink term.

Again, Equation(4) tells us that when $\xi<\xi_{i}$, there is a balance between the heat convected from the boundary $\xi_{i}$ to a control volume in the interior, heat conducted out of the control volume and the heat generated in the control volume due to the source, $a_{i}(\eta)$.

From Equations (3) and (4), it can be seen that for a given convective heat flux (given number of grid lines), as the product, $a_{i} b_{i}$, decreases, the heat transfer coefficient decreases proportionally in magnitude which means that the temperature gradient at the boundary $\xi_{i}$ has increased so that $\xi$ approaches $\xi_{i}$ rapidly. This means that there is a large gradient in $\xi$ from the grid boundary $i$ to the interior, thereby resulting in a highly clustered grid near the boundary.

Away from this grid boundary, $b_{i}\left|\xi-\xi_{i}\right|$ or $b_{i}\left|\eta-\eta_{i}\right|$ is large, and we are left with the Laplace equation, $\Delta \xi=0$ or $\Delta \eta=0$. Extremum principle is unconditionally maintained there, since the solution is harmonic in this case.

Referring to Equations (3) and (4), the Green's Theorem gives us respectively,

$$
\begin{aligned}
\iint_{S}\left(-a_{i}+a_{i} b_{i} \theta\right) d \sigma & =\int_{C} \partial_{n} \theta d s \\
\iint_{S}\left(a_{i}+a_{i} b_{i} \theta\right) d \sigma & =\int_{C} \partial_{n} \theta d s
\end{aligned}
$$

where $\mathrm{S}$ is the surface area of a closed domain, $\mathrm{C}$ is the boundary enclosing this domain, $\mathrm{n}$ is the normal to the surface, $d \sigma$ is the elemental area and ds is an elemental arc.

The integrands on the left hand side, $\pm a_{i}$ and $a_{i} b_{i} \theta$ represent the heat sink/source term and the convection term respectively, and the integrand on the right hand side represents the heat flux through the boundary $\mathrm{C}$.

Equations (5) and (6) are used as constraints to fix $b_{i}$ uniquely for a solution consistent with the specification of the boundary data. The extremum principle will be satisfied at the ith boundary, which is the requirement in the grid generation problems, since the energy conservation principle is satisfied. The term, $\pm a_{i}(\eta)$, respectively in Eqs. (6) and (5), is calculated iteratively through the 
solution process, which together with $b_{i}$ ensures the grid orthogonality and a given grid spacing at the ith grid boundary.

In the design of the inhomogeneous terms, $P(\xi, \eta)$ and $Q(\xi, \eta)$ in Eqs. $1(\mathrm{a})$ and $1(\mathrm{~b})$, there is no restriction on the nature of the sink/source term, $\pm a_{i}$. It can change sign which indicates the presence of sources and sinks, subject to the constraint given by Equations (5) and (6). Otherwise, improper combinations of sources and sinks will violate the extremum principle. If over the domain, there is a net rate of heat generation due to the source/sink combination, then there has to be a positive heat flux convected away and vice-versa. This requirement will automatically be satisfied by Equations (5) and (6).

If there is a point heat source present in the domain, the isothermals (temperature or $\xi$ or $\eta$ contour lines) will tend to cluster around it since the gradients in the vicinity of the source will be positive toward the source and high, depending upon the strength of the source, and conversely for a heat sink. Same argument applies to a line heat source and sink. By analogy, if the source term turns out to be positive over some parts of the domain, then the curvilinear coordinate lines will tend towards lines with higher coordinate values and vice-versa.

\section{Solution Procedure}

First, the boundary data are selected appropriate to the physics of the problem, so that the gradients in physical quantities can be resolved adequately. Since there is a symmetry plane and a rotational symmetry present in the gear problem, the half-tooth grid is reflected about this symmetry plane and then the tooth grid is rotated about a moving axis of periodicity, thus substantially reducing the computational effort in prescribing the initial distribution of the entire 19-tooth gear at time, $t=0$. But, for the elliptic grid generation of the entire gear after this inital time, periodic boundary conditions are used in the circumferential direction.

Then, by interchanging the independent and dependent variables, the governing equations to be solved in the computational space $(\xi, \eta)$ become

$$
\begin{aligned}
& \alpha x_{\xi \xi}-2 \beta x_{\xi \eta}+\gamma x_{\eta \eta}=-J^{2}\left(P(\xi, \eta) x_{\xi}+Q(\xi, \eta) x_{\eta}\right) \\
& \alpha y_{\xi \xi}-2 \beta y_{\xi \eta}+\gamma y_{\eta \eta}=-J^{2}\left(P(\xi, \eta) y_{\xi}+Q(\xi, \eta) y_{\eta}\right)
\end{aligned}
$$

These equations are solved in the computational space using a line SOR relaxation algorithm where each coordinate line in one curvilinear coordinate direction is solved semi-implicitly using the Thomas algorithm for tri-diagonal systems. The inhomogeneous terms referred to above are designed and incorporated so that a desired grid behavior near the boundaries is achieved.

The inhomogeneous problem is solved using a technique similar to that of Ref. 3 by over-relaxing the inhomogeneous terms during the iteration process. The inhomogeneous terms used in Ref. 3 are well suited for external boundary value problems where they allow for clustering in only one curvilinear coordinate direction, normal to the body. But, in internal boundary value problems, inhomogeneous terms have to take account of the influence of the boundaries in both curvilinear coordinate directions. The inhomogeneous terms used here allow for clustering in both coordinate directions.

The inhomogeneous terms, $P(\xi, \eta)$ and $Q(\xi, \eta)$, are evaluated at the boundaries in terms of the left hand side at each line relaxation sweep. Then outward from each boundary, the inhomogeneous terms are attenuated through an exponential function in each direction, as discussed above. In $\xi$ direction, outward from a given $\xi_{i}$ boundary, this exponential term is of the form, $-a_{i} \exp \left(-b_{i}\left|\xi-\xi_{i}\right|\right)$, and, in $\eta$ direction, it is of the form, $-a_{i} \exp \left(-b_{i}\left|\eta-\eta_{i}\right|\right)$.

In what is stated above, a proof of concept study for an annulus and then a gear tooth is carried out to demonstrate the usefulness of the new boundary constraints. Then, the grid for the complete 19-tooth gear is generated using periodic boundary conditions in the circumferential direction. This is the only way to solve the grid generation problem in a larger context of structural dynamical simulation of the gear, since the grid for the entire gear will be subjected to dynamic stresses nonuniformly.

The boundary constraints given by Equations (5) and (6) are applied to a region close to the boundary. For example, a finite slender strip close to the boundary, $\eta_{\max }$, as shown in Fig. 1, is used as a control volume to evaluate the heat source term and the convective flux term over it with the net 
heat flux calculated around it. At the $\eta=\eta_{\max }$ boundary; the heat transfer coefficient, $h_{2}$ is large, whereas the heat transfer coefficient, $h_{1}$ is moderate. The pin fin is thin in the $z$ direction, and the ambient enveloping the fin in the neighborhood of and around the $\eta_{\max }$ boundary can be imagined to be at a temperature, $\eta_{\infty}=\eta_{\max }$, since $h_{2}$ is large. Now, as $h_{1}$ becomes smaller, the temperature gradient in the y direction near the boundary $\eta_{\max }$ increases, for a given heat flux (given number of grid lines, per the boundary prescription), and therefore the clustering there becomes denser. This is the physical basis for the argument that the clustering at a given boundary becomes denser when the decay parameter, $b_{i}$, associated with the $a_{i} \dot{b}_{i} \hat{\theta}$ term in Eq. 4 decreases.

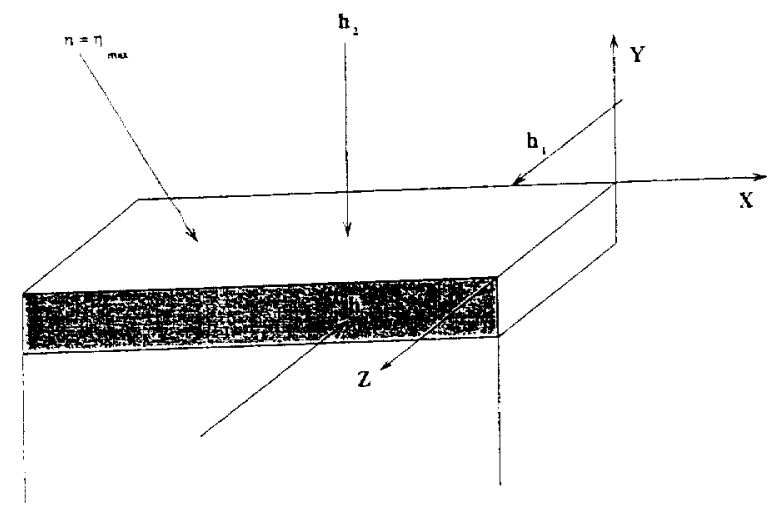

Figure 1: A schematic showing the finite slender strip over which the boundary constraint is applied

\section{Results}

The new boundary constraints were validated by generating two-dimensional interior grids for an annulus and a gear tooth. Figure 2 shows an interior grid for an annulus, clustered at two opposing boundaries in, e.g., the $\xi$ direction. The degree of clustering at/near the $\eta$ boundaries, e.g., is measured by a clustering parameter, $\Delta S / \Delta S_{a v}$, where $\Delta S$ is the normal spacing at the $\eta$ boundary and $\Delta S_{a v}$ is the average spacing based on the length of the $\xi$ coordinate line from one $\eta$ boundary to the opposing $\eta$ boundary and the number of grid points along this $\xi$ coordinate line. Therefore, as the clustering parameter at a given boundary decreases in value, clustering becomes denser at/near that boundary.

Figs. $3 \mathrm{a}$ and $3 \mathrm{~b}$ show the lower boundary decay parameter convergence rate corresponding to the clustering parameter value of 0.1 , and Figs. $3 c$ and $3 \mathrm{~d}$ show the corresponding rate for the upper boundary. The ordinate for these figures shows the number of iterations and the abscissa the value of the decay parameter. The calculations are stopped when the relative convergence criterion that the average fnal spacing at any given boundary is within 10the average initial spacing at that boundary.

Again, as is seen, the decay parameter converges to a stable value rapidly. Corresponding to this converged solution, the decay functions for the lower and upper boundaries are shown in Figs. 4a and $4 \mathrm{~b}$. The ordinate in these figures shows the number of grid points along the $\xi$ direction between the top and bottom $\eta$ boundaries.

In Fig. 5, a grid for the gear tooth corresponding to the clustering parameter value of 0.1 is shown. Figs. 6 a to $6 \mathrm{~d}$ show the results for the decay parameter convergence corresponding to this grid. The corresponding decay function distribution is shown in Fig. 7.

In Fig. 8, a grid for the gear tooth corresponding to the clustering parameter value of 0.05 is shown. Figs. $9 \mathrm{a}$ to $9 \mathrm{~d}$ show the corresponding results for the decay parameter convergence. Figure 10 shows the corresponding results for the decay function distribution for the lower and upper boundaries. 


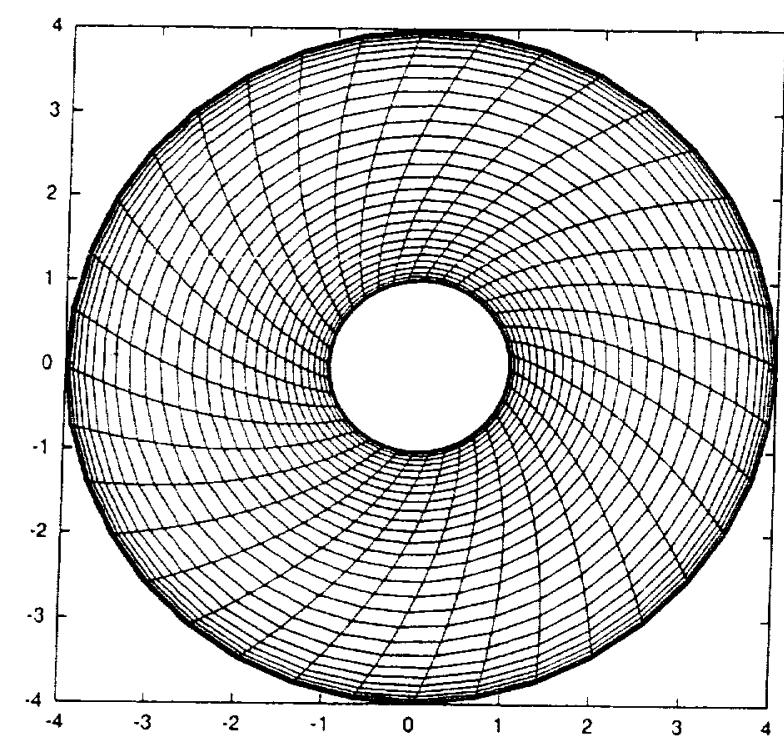

Figure 2: Finite difference grid of annulus, clustering parameter $=0.1$

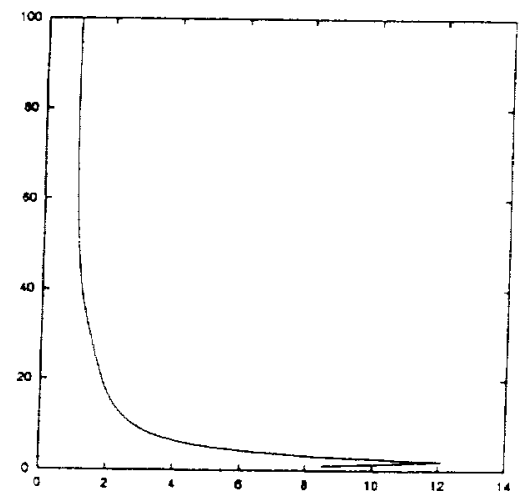

(a) 100 iterations

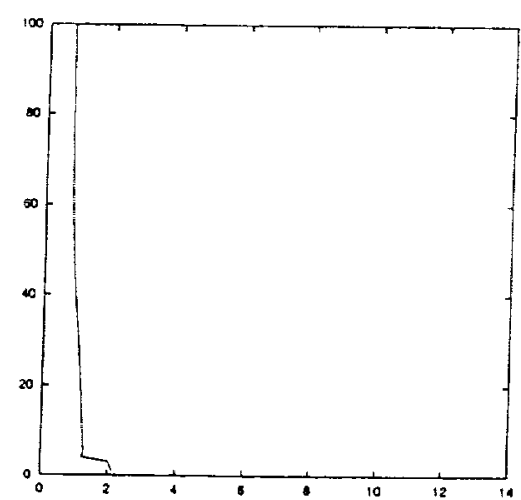

(c) 100 iterations

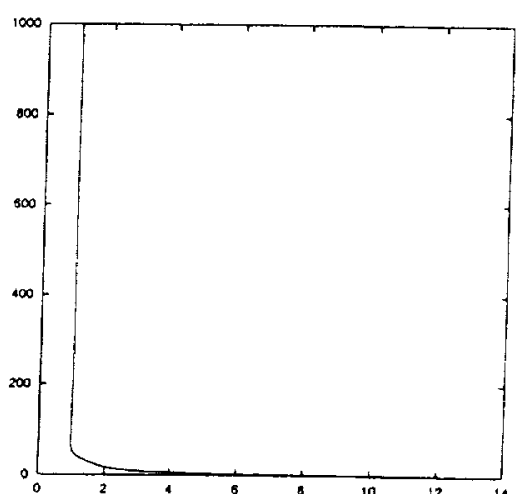

(b) 1000 iterations

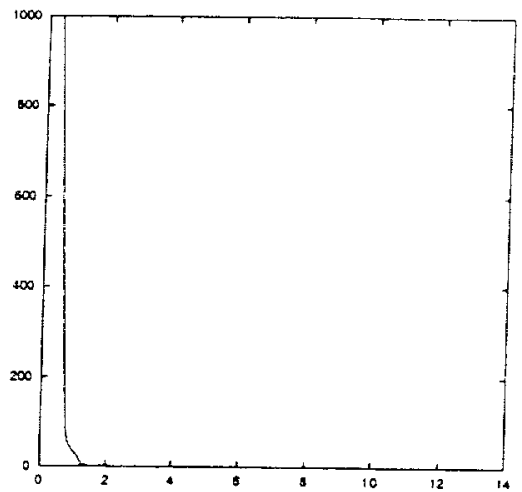

(d) 1000 iterations

Figure 3: Results for the annulus: (a), (b) Lower boundary decay parameter convergence, (c), (d) Upper boundary decay parameter convergence. 


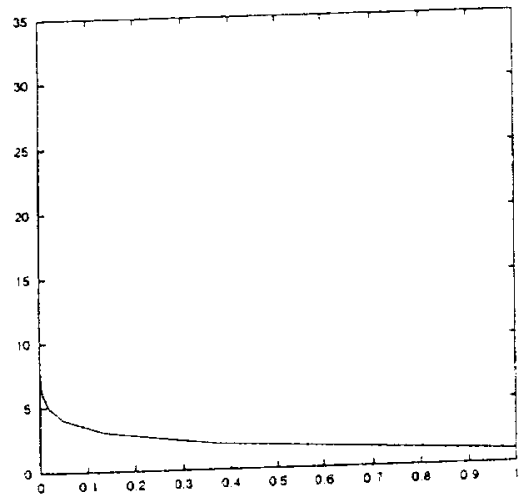

(a)

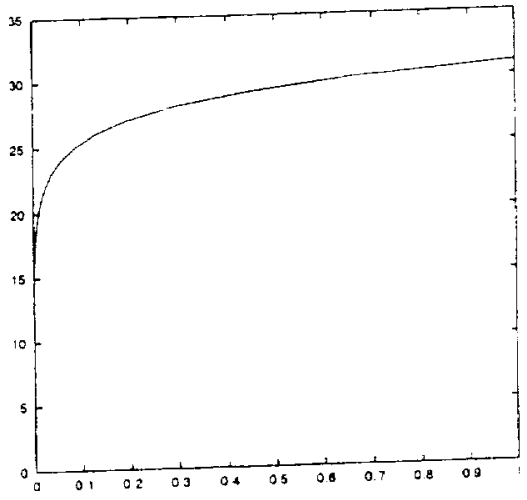

(b)

Figure 4: Results for the annulus: a) Lower boundary decay function distribution, b) Upper boundary decay function distribution.

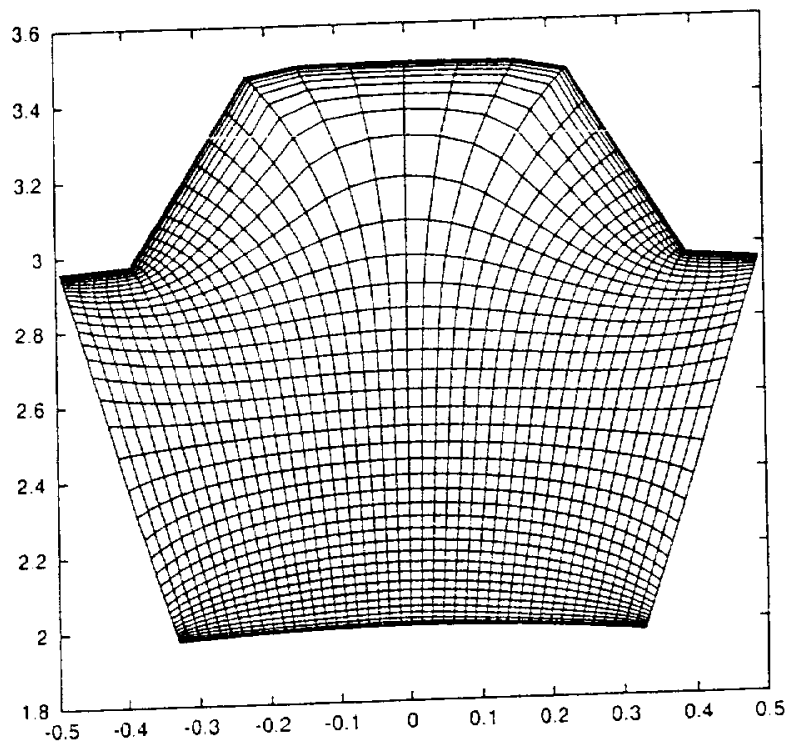

Figure 5: Finite difference grid of pinion gear tooth, clustering parameter $=0.1$ 


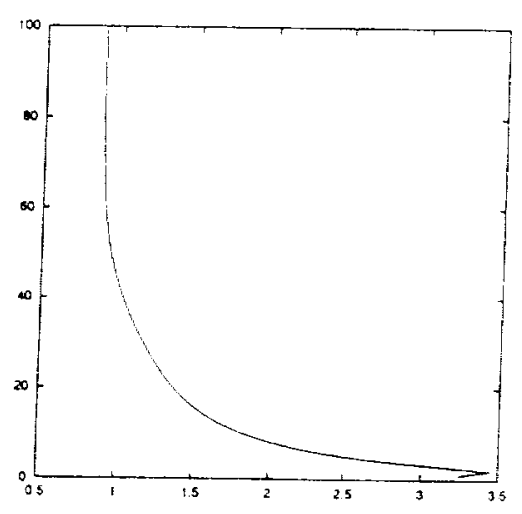

(a) 100 iterations

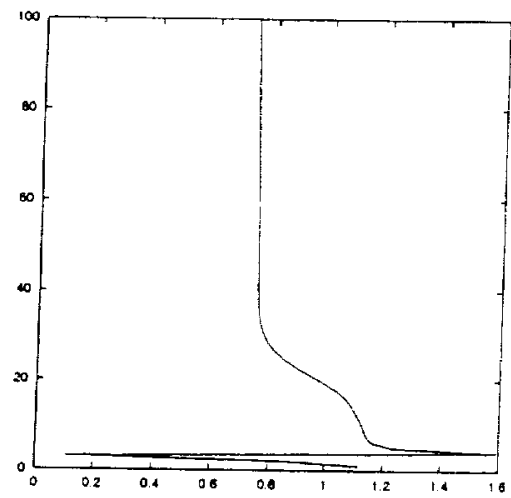

(c) 100 iterations

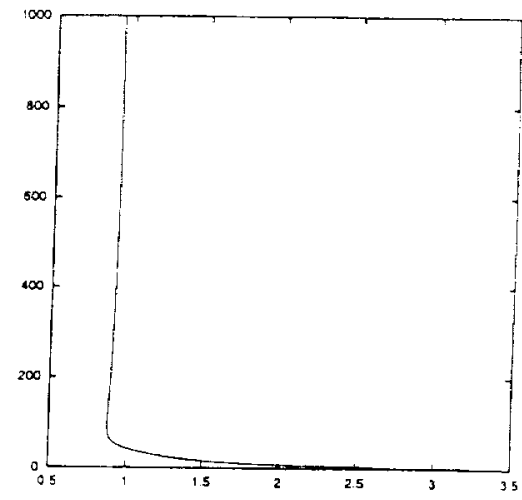

(b) 1000 iterations

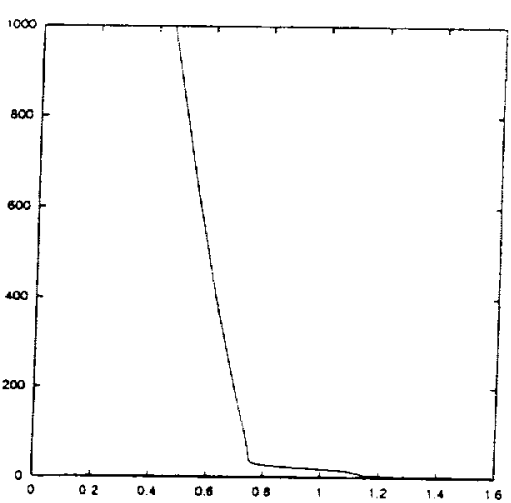

(d) 1000 iterations

Figure 6: Results for the gear tooth, clustering parameter of 0.1: (a), (b) Lower boundary decay parameter convergence, (c), (d) Upper boundary decay parameter convergence.

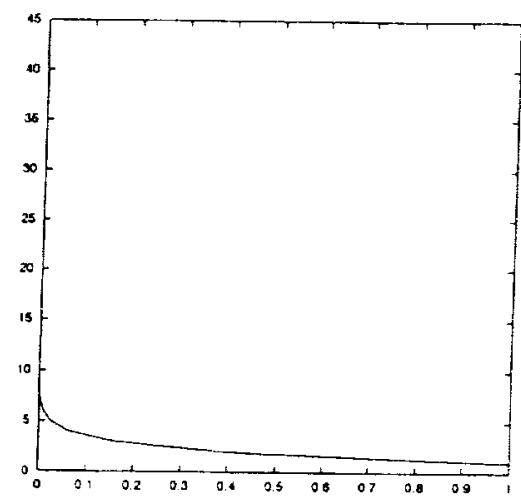

(a)

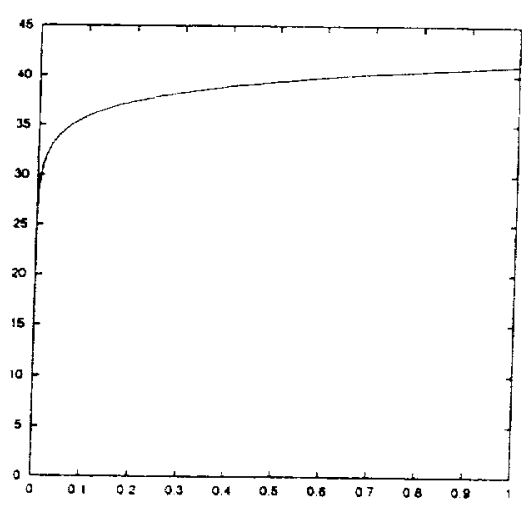

(b)

Figure 7: Results for the gear tooth, clustering parameter of 0.1 : a) Lower boundary decay function distribution, b) Upper boundary decay function distribution. 


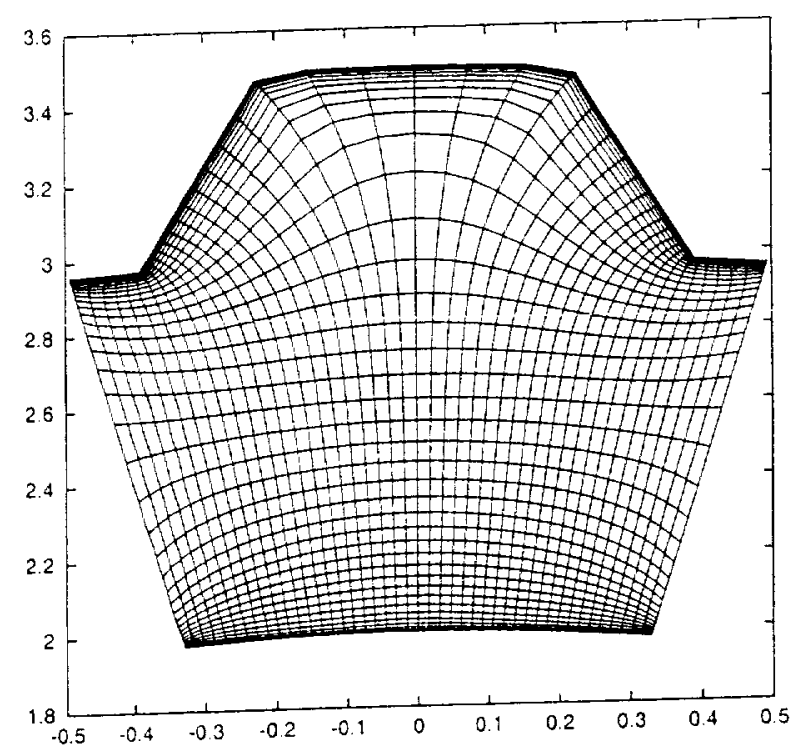

Figure 8: Finite difference grid of pinion gear tooth, clustering parameter $=0.05$

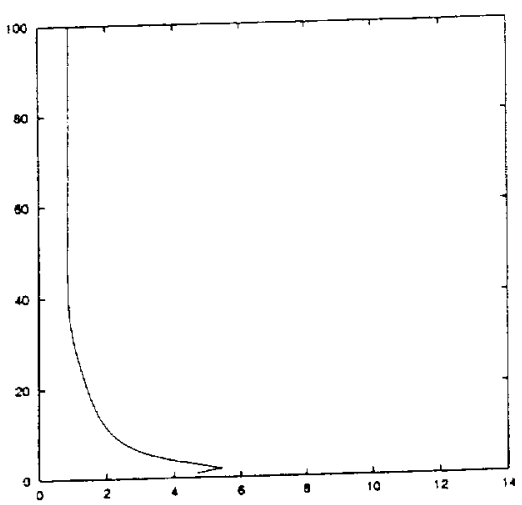

(a) 100 iterations

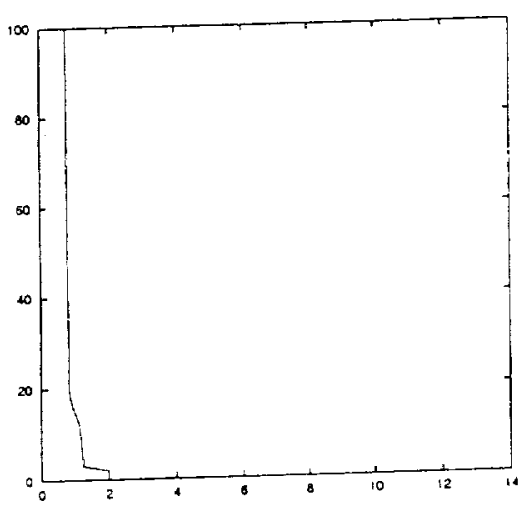

(c) 100 iterations

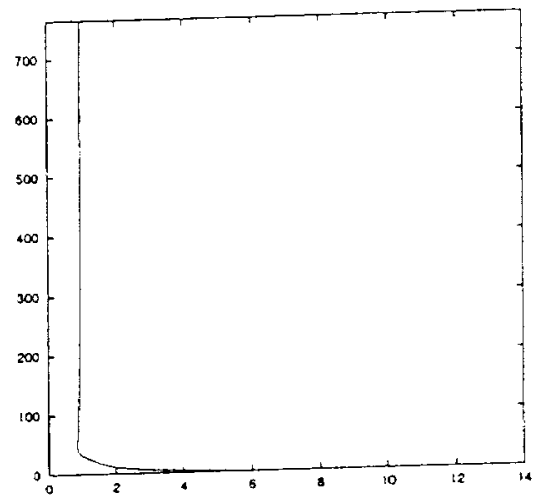

(b) 700 iterations

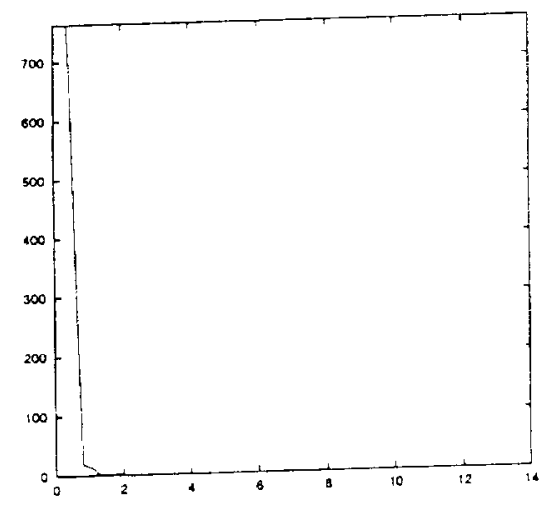

(d) 700 iterations

Figure 9: Results for the gear tooth, clustering parameter of 0.05: (a), (b) Lower boundary decay parameter convergence, (c), (d) Upper boundary decay parameter convergence. 


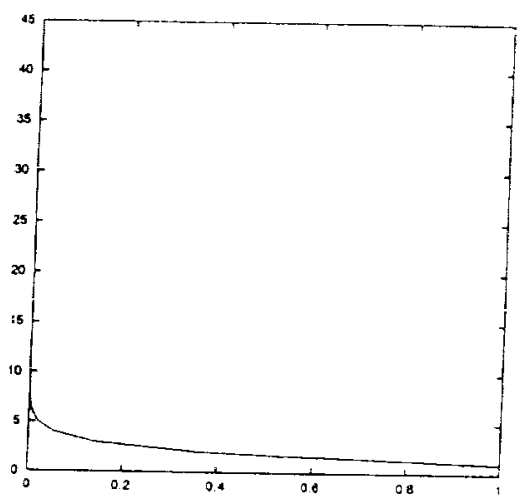

(a)

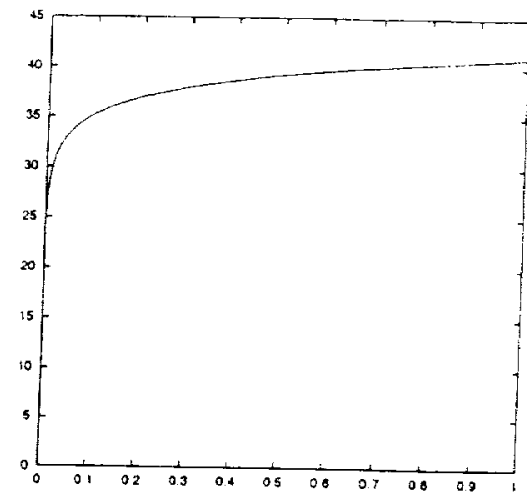

(b)

Figure 10: Results for the gear tooth, clustering parameter of 0.05: a) Lower boundary decay function distribution, b) Upper boundary decay function distribution.

As can be observed by comparing with Figs. 5 and 6 corresponding to clustering parameter of 0.1 , the degree of clustering has increased at/near the boundaries, and therefore the decay parameter value has also correspondingly decreased when the clustering parameter has decreased from 0.1 to 0.05 .

Figure 11 shows a section of the complete 19-tooth gear grid which was generated using periodic boundary conditions in the $\xi$ direction (circumferential direction) with a clustering parameter of
0.05 .

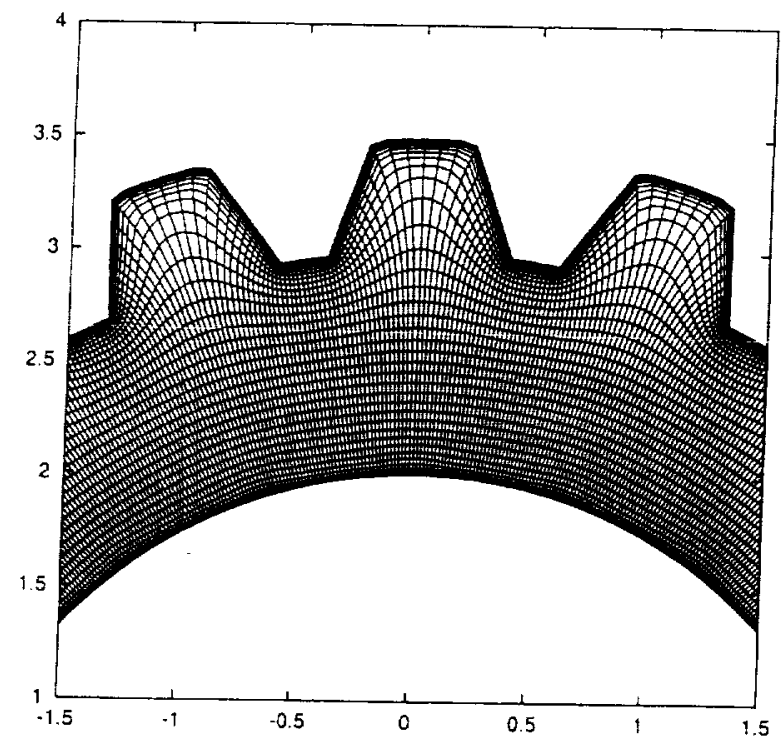

Figure 11: Section of a finite difference grid of pinion gear, clustering parameter $=0.05$

The grid is seen to be smooth and orthogonal throughout in this case, as expected. Figure 12 shows the gear tooth grid for one-sided clustering at the $\eta_{\max }$ boundary. The value of the clustering parameter at the lower boundary is 5.0, which means that the grid is coarsest at the lower boundary and it progressively gets refined toward the upper boundary. Dirichlet boundary conditions were applied at the $\xi_{1}$ and $\xi_{\max }$ boundaries. The strict enforcement of orthogonality at these boundaries is not of concern here since the grid for the 19-tooth gear is generated with the periodic boundary conditions prescribed at these boundaries. Fig. 12 is another test case showing the efficacy of the new constraints derived here. 


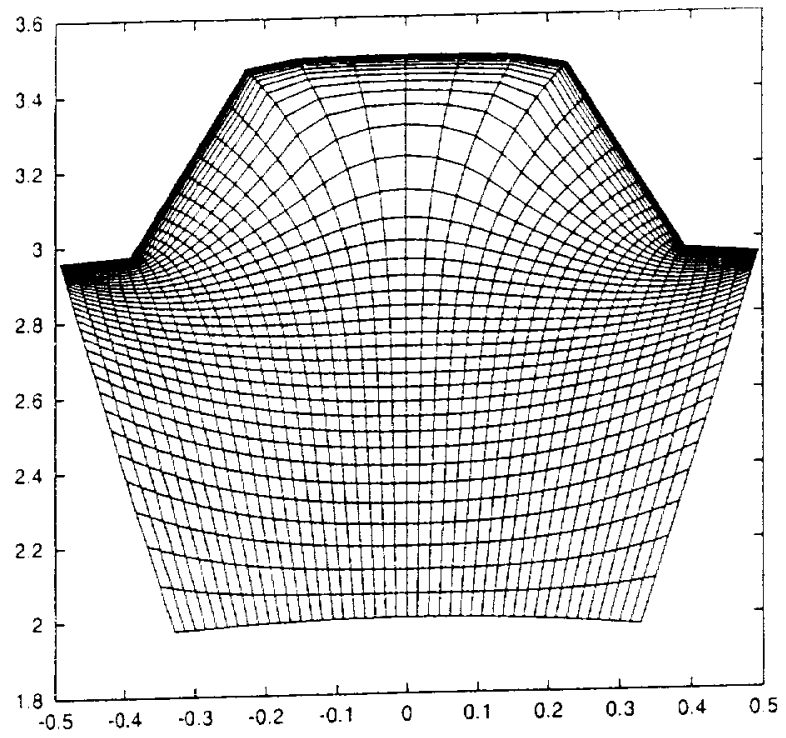

Figure 12: Finite difference grid of pinion gear tooth, clustering parameter $=5.0$

Figures $13 \mathrm{a}$ and $13 \mathrm{~b}$ show the corresponding lower boundary decay parameter convergence rate, and Figs. 13c and 13d show the corresponding rate for the upper boundary. Here also, the decay parameter converges to a stable value rapidly.

Corresponding to the converged solution, the decay functions for the lower and upper boundaries are shown in Figs. 14a and 14b. The difference here from the previous two-sided clustering is that the clustering at lower boundary, $\eta_{1}$, is sparse and that at the upper boundary, $\eta_{\max }$, is dense as desired. Accordingly, the decay parameter at the lower boundary is large and that at the upper boundary is small. The new boundary constraints in this case also yield a grid properly clustered at both the boundaries.

Another grid simulation with the same clustering parameter value of 5.0 was made with the complete 19-tooth gear. Periodic boundary conditions in the circumferential direction were used in this case. The corresponding grid is shown in Fig. 15. The slit $A A^{\prime}$ represents the radial line where periodic boundary conditions are enforced in the circumferential direction.

As a comparative example, a test case was run with an a priori prescribed value of the decay parameter of 0.15 corresponding to the case shown in Fig. 12, i.e., with a clustering parameter value of 5.0, and the resulting grid rapidly degrades as shown in Fig. 16(a) and the corresponding solution convergence history is shown in Fig. 16(b), where after 50 iterations, the solution aborts due to negative values of Jacobians. This is just an example of the problems encountered in the trial-and-error process in prescribing the decay parameter manually, for which now a solution has been found through the new constraints derived in the present study.

Finally, figure 17 shows a finite-difference grid model of pinion and driven gears in mesh. 


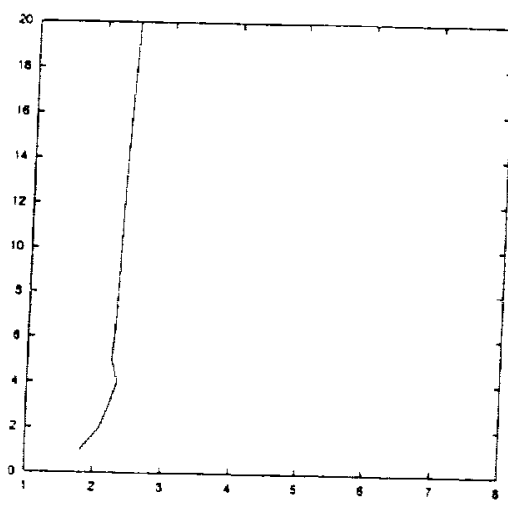

(a) 20 iterations

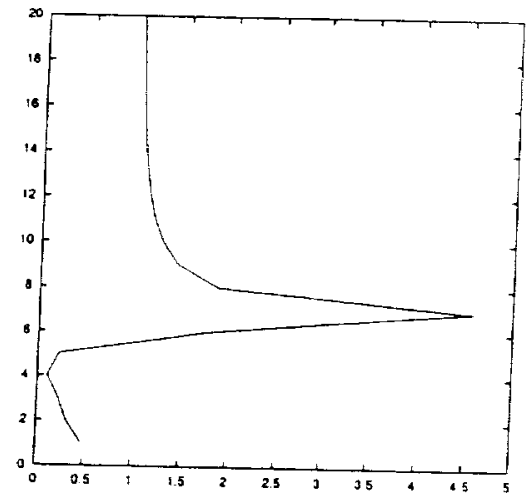

(c) 20 iterations

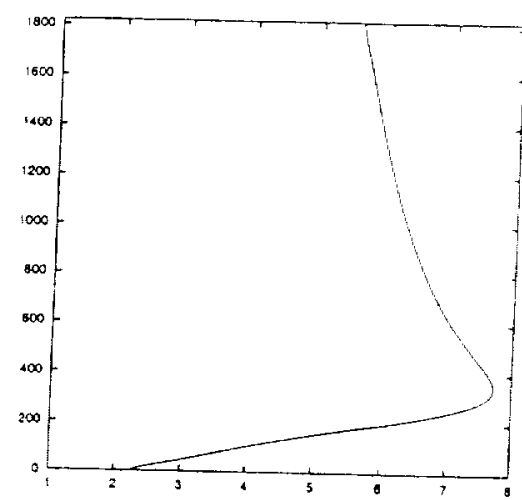

(b) 1800 iterations

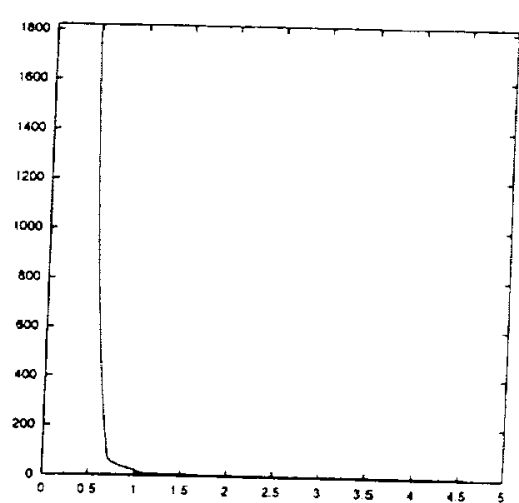

(d) 1800 iterations

Figure 13: Results for the gear tooth, lower boundary clustering parameter of 5.0: (a), (b) Lower boundary decay parameter convergence, (c), (d) Upper boundary decay parameter convergence.

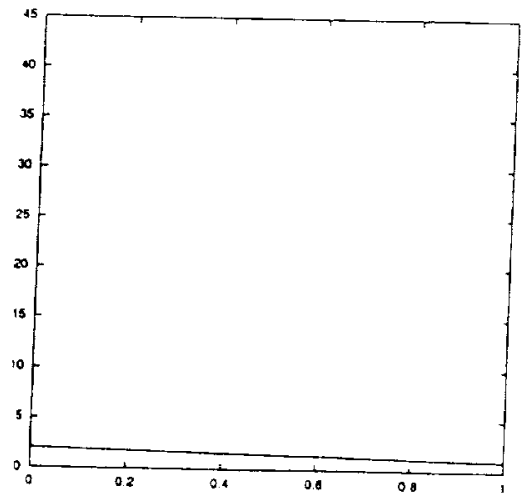

(a)

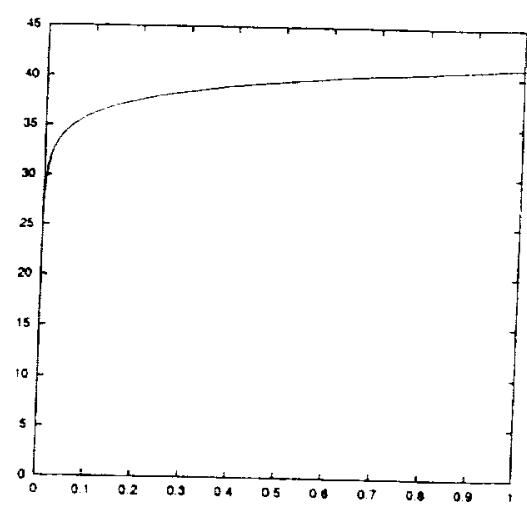

(b)

Figure 14: Results for the gear tooth, lower boundary clustering parameter of 5.0: a) Lower boundary decay function distribution, b) Upper boundary decay function distribution. 


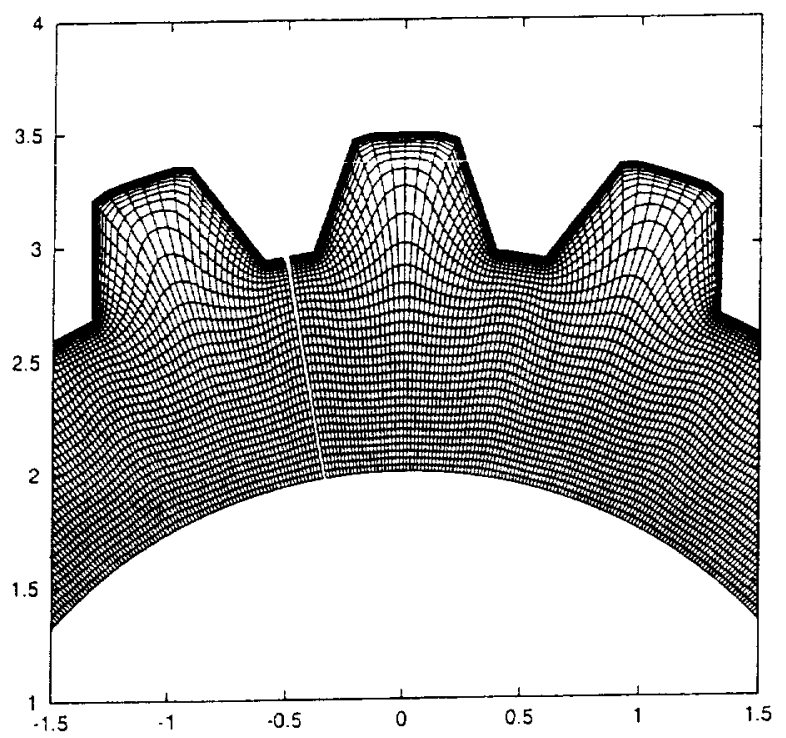

Figure 15: Finite difference grid of a section of pinion gear, clustering parameter $=5.0$

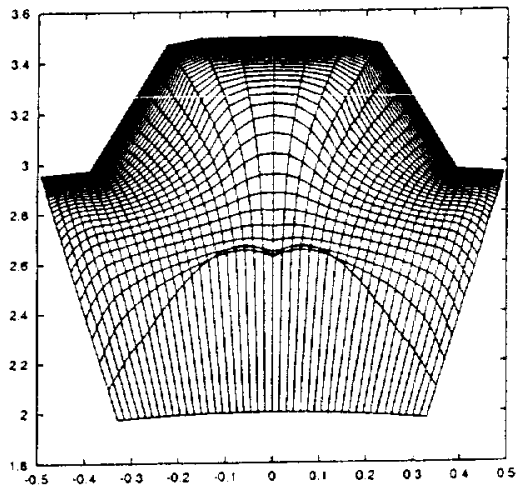

(a) Negative Jacobians

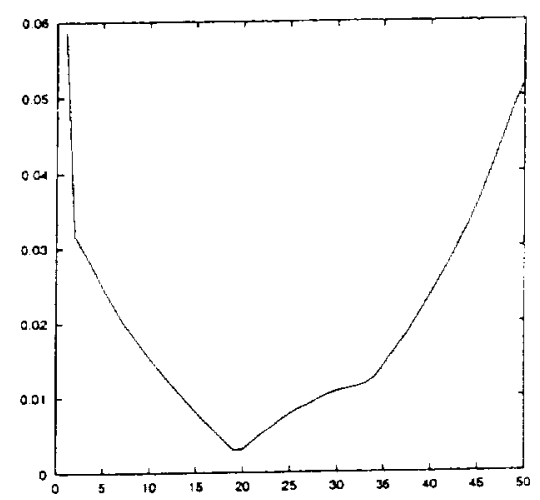

(b) Solution diverges rapidly

Figure 16: Comparative results for the gear tooth with a fixed decay parameter value of 0.15 ; clustering arameter of 5.0: a) grid for the tooth b) convergence history. 


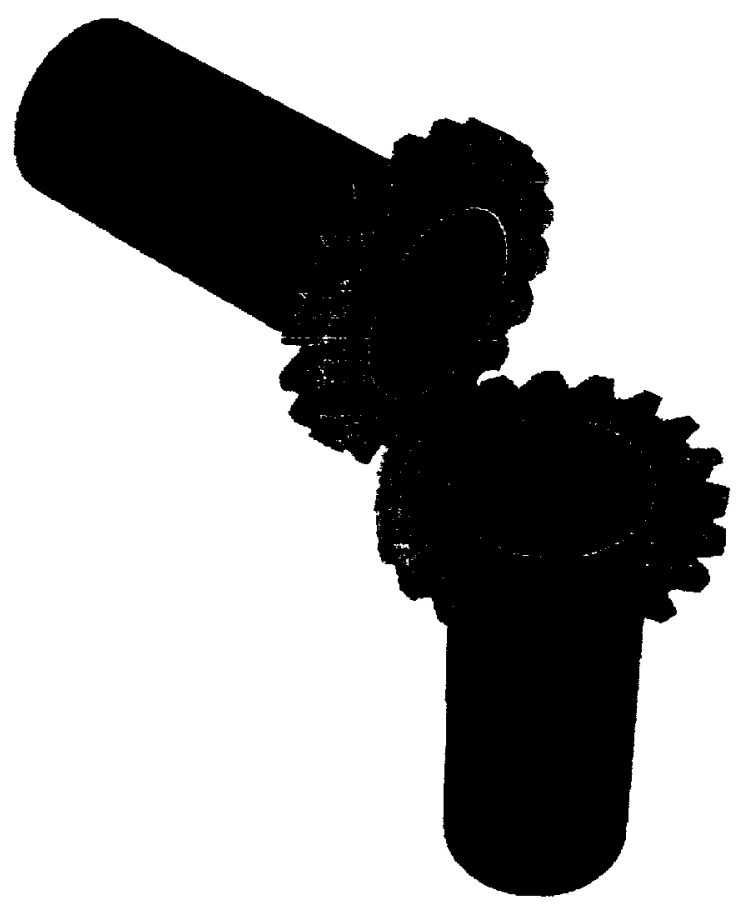

Figure 17: Finite difference grid model of pinion and driven gears in mesh

\section{Concluding Remarks}

The boundary constraints for elliptic grid generation problems developed in this study have been demonstrated for two internal geometrical configurations. Internal grids for an annulus, a gear tooth and a complete 19-tooth gear were generated using the new constraints. Smooth clustered grids have been generated using these constraints without hit-and-trial prescription of decay parameters and without any recourse to redistribution of grid points, which has been a common approach used in elliptic grid generation problems until now. With new constraints, elliptic grids can be generated in simulation time without any manual intervention thus making problems of structural dynamics and fuid dynamics over compliant boundaries straightforward. Thus, a fully automated elliptic grid generation methodology has been developed and validated.

\section{REFERENCES}

1. Thompson, J. F., Thames, F. C. and Mastin, C. W., "Automatic Numerical Generation of Budy-Fitted Curvilinear Coordinate System for Field Containing Any Number of Arbitrary TwoDimensional Bodies," J. Computational Physics, Vol. 15, pp. 299-319, 1974

2. Thompson, J. F., Thames, F. C. and Mastin, C. W., "TOMCAT - A Code for Numerical Generation of Boundary-Fitted Curvilinear Coordinate Systems on Fields Containing Any Number of Arbitrary Two-Dimensional Bodies," J. Computational Physics, Vol. 24, pp. 274-302, 1977

3. Steger, J. L. and Sorenson, R. L., "Automatic Mesh-Point Clustering Near a Boundary in Grid Generation with Elliptic Partial Differential Equations," J. Computational Physics, Vol. 33, pp. $405-410,1979$

4. Jeng, Y. N. and Kuo, W. J., "Two-Dimensional Elliptic Grid Solver Lising Boundary Grid Control and Curvature Correction," AIAA J., Vol. 38, No. 2, February 2000 
5. Kaul, L. K. and Huff, Edward M., "Automated Gear Teeth Grid Generation via Solution of Elliptic Pdes,"SIA.M Conference on Geometric Design and Computing, Nov. 5-8, 2001, Sacramento, C.A

6. Kaul, U. K. and Huff, Edward M., "Elliptic Grid Generation of Spiral-Bevel Pinion Gear Typical of OH-58 Helicopter Transmission," NASA TM-2002-210932, February 2002

7. Kaul, C. K. and Chaussee, D. S., "A Comparative Study of the Parabolized Navier-Stokes (PNS) Code Using Various Grid Generation Techniques," International J. Computers and Fluids, Vol. 13, No. 4, pp 421-441, 1985 
- 